\title{
Determination of parameters and stability zones of pendulum auto-balancer of rotor, installed in housing on elastic supports
}

\author{
A.I. Artyunin \\ Irkutsk State Transport University \\ Irkutsk, Russia \\ e-mail: artyunin_ai@irgups.ru
}

\author{
S.V. Eliseev \\ Irkutsk State Transport University \\ Irkutsk, Russia
}

\author{
O.Y. Sumenkov \\ Tomsk Polytechnic University \\ Tomsk, Russia
}

\begin{abstract}
Using the methods of the small parameter of nonlinear mechanics, the formulas for determining the parameters of the pendulum auto-balancer were obtained in the article. Conditions for the stability of the auto-balancing process of the unbalanced rotor, fastened in the housing on the elastic supports, were also found. It is established that the stable operation of the auto-balancer can be implemented in the angular velocity ranges of the rotor due to the choice of the stiffness characteristics of the elastic supports.
\end{abstract}

Keywords-Housing, Rotor, Auto-balancer, Pendulum, Stability, Elastic supports)

\section{INTRODUCTION}

The main reason for increasing the dynamic loading and increasing the vibration level of machines and mechanisms with rotating rotors is a change in the imbalance of the rotors during operation. The change in the imbalance is due to wear on the bearings and rotor assemblies, weakening of joints and fastenings, deformation of the rotor elements, adhesion of the processed material and other reasons. This operational change in the imbalance of the rotor is usually at a low speed and is random. In this connection, it appears expedient to use automatic balancing devices that compensate for the change in the imbalance of the rotor in operating conditions without interrupting the technological process and working according to the principles of self-centering.

The most complete description of automatic balancing devices, their advantages and disadvantages are given in the monograph by A.A. Gusarov [1]. Of great importance for the development of the theory of automatic balancing of rotors are the works of I.I. Blekhman [2], [3]. These works lay down the methodological bases for calculating automatic balancing devices and formulate a generalized principle of autobalancing, based on the method of small parameter and the method of separation of motion of nonlinear mechanics. Methods for calculating autobalancing devices of rotor systems with a large number of degrees of freedom were developed in the works of V.P. Nesterenko [4] and A.I. Artyunin [5] on the basis of these representations.

A significant contribution to the solution of scientific and practical problems of automatic balancing of rotors was made by G.B. Filimonikhin [6]. Various problems of the dynamics of rotors with autobalancers have been studied in [7-15]. The current state of the problem of balancing rotors is presented quite fully in the article of A.N. Nikiforov [16]. In connection with the chosen direction of research in this article, one should mention the works [17], [18], related to the study of the autobalancing process of rotors installed in the housing.

\section{DESCRIPTION OF THE MOVEMENT OF THE DYNAMIC ROTOR MODEL IN THE HOUSING WITH PENDULUMS MOUNTED ON THE ROTOR FOR AUTO- BALANCING}

For the study, let us select and use the dynamic model of the rotor system with an auto-balancer shown in Fig. 1. This model is a massive housing, fastened on a fixed base with weightless elastic supports. In the housing a rigid rotor is installed in its own bearings and rotates with an angular velocity $\omega$. Two pendulums of the same mass $m$ and length $l$ are suspended on the rotor shaft in pairs on both sides of the rotor to compensate for the dynamic imbalance from possible free rotation.

The motion of the model will be considered with respect to the fixed coordinate system Oxyz. Since in most real constructions there is practically no motion of the housing along the axis of the rotor, then, to describe the motion of the rotor with the housing, we choose the following generalized coordinates: y, z are linear displacements of the point $O_{1}\left(O_{1}\right.$ is the point of intersection of the rotor axis with the plane passing through its center of mass perpendicularly axes of rotation, $\varphi_{\mathrm{x}}, \theta, \psi$ are the angular movements of the housing 
along with the rotor around axes $x_{1}, y_{1}, z_{1}$. Axes $x_{1}, y_{1}, z_{1}$ are parallel to axes $x, y, z$ at the initial moment of time. The positions of the pendulums are determined by angles $\varphi_{1}, \varphi_{2}$, $\varphi_{3}, \varphi_{4}$. The positive directions for reading these angles are shown in Fig. 1.

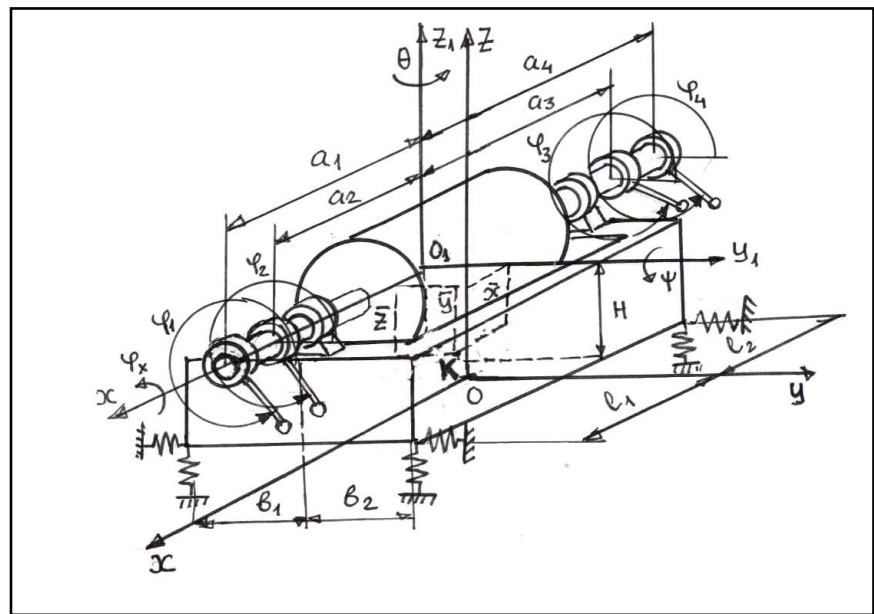

Fig. 1. A dynamic model of the rotor with pendulum auto-balancers, installed in the housing on elastic supports.

We accept some assumptions that negatively affect the final result. First, we will not take into account the damping due to its little influence on the choice of the parameters of the auto-balancer and on the position of the stability zones. The resistance to rotation of the pendulums will be proportional to their relative rotational velocities. Secondly, suppose that the main axes of inertia of the housing are parallel to the axes $x_{1}$, $y_{1}, z_{1}$. Third, we assume that the housing supports are isotropic, and the distance between the pendulum suspension points in each pair is small, compared to the distance from the center of the rotor masses to the pendulum suspension points. We take the following notation: $M_{p}, A, C$ are mass, equatorial and polar moments of inertia of the rotor; $M_{k}, I_{\mathrm{x}}, I_{\mathrm{y}}, I_{\mathrm{z}}$ are mass and moments of inertia of the housing; $e, \delta, \varepsilon$ are parameters of the rotor imbalance: $c_{1 y}=c_{2 y}=c_{3 y}=c_{4 y}=c_{y}$; $c_{1 z}=c_{2 z}=c_{3 z}=c_{4 z}=c_{z}$ are stiffness coefficients of 4 horizontal and 4 vertical supports of the housing; $\bar{x}, \bar{y}, \bar{z}$ are constant coordinates of the center of mass of the hull in reference system $x_{1} y_{1} z_{1} ; a_{1}=a_{2}=a_{3}=a_{4}=a$ are distances from the center of mass of the rotor to the pendulum suspension points; $\beta_{\mathrm{O}}$ is the coefficient of resistance to rotation of the pendulums. We proceed further from the assumption that the linear and angular displacements of the housing with the rotor are small in the sense that linear differential equations are sufficient for describing their motion without pendulums. Then the equations of motion of the model under study are divided into equations for the rotor with the housing:

$$
[M]\{\ddot{q}\}+[G]\{\dot{q}\}+[K]\{q\}=\{F\}
$$

and equations for the auto-balancer pendulums:

$$
\begin{aligned}
& \ddot{\varphi}_{k}+\beta_{O}\left(\dot{\varphi}_{k}-\omega\right)=\left[\ddot{y} \sin \varphi_{k}-\ddot{z} \cos \varphi_{k}+\right. \\
& \left.+\alpha \sigma_{k}\left(\ddot{\theta} \sin \varphi_{k}-\ddot{\psi} \cos \varphi_{k}\right)-g \cos \varphi_{k}\right] / l,
\end{aligned}
$$

where $\{q\}=\left\{y, z, \theta, \psi, \varphi_{x}\right\}^{T} ; \quad ; k=\overline{1,4}$;

$$
\begin{gathered}
{[M]=\left|\begin{array}{ccccc}
M^{*} & 0 & m_{1} & 0 & m_{2} \\
0 & M^{*} & 0 & m_{1} & m_{3} \\
m_{1} & 0 & I_{z}^{*} & m_{4} & m_{5} \\
0 & m_{1} & m_{4} & I_{y}^{*} & m_{6} \\
m_{2} & m_{3} & m_{5} & m_{6} & I_{x}^{*}
\end{array}\right| ;} \\
{[G]=\left|\begin{array}{ccccc}
0 & 0 & 0 & 0 & 0 \\
0 & 0 & 0 & 0 & 0 \\
0 & 0 & 0 & C \omega & 0 \\
0 & 0 & -C \omega & 0 & 0 \\
0 & 0 & 0 & 0 & 0
\end{array}\right| ;} \\
{[K]=\left|\begin{array}{ccccc}
c_{1} & 0 & c_{2} & 0 & c_{1} H \\
0 & c_{3} & 0 & c_{14} & c_{5} \\
c_{2} & 0 & c_{6} & 0 & c_{2} H \\
0 & c_{4} & 0 & c_{7} & c_{8} \\
c_{1} H & c_{5} & c_{2} H & c_{8} & c_{9}
\end{array}\right| .}
\end{gathered}
$$

When compiling the matrices $[M],[K]$ the following notation was used:

$$
\begin{aligned}
& M^{*}=M_{p}+M_{k}+4 m ; \\
& I_{z}^{*}=A+M_{k}\left(\bar{x}^{2}+\bar{y}^{2}\right)+I_{z}+m \sum_{k=1}^{4} a_{k}^{2} ; \\
& I_{y}^{*}=A+M_{k}\left(\bar{x}^{2}+\bar{z}^{2}\right)+I_{y}+m \sum_{k=1}^{4} a_{k}^{2} ; \\
& I_{x}^{*}=C+M_{k}\left(\bar{y}^{2}+\bar{z}^{2}\right)+I_{x} ; \\
& m_{1}=\bar{x} M_{k} ; \quad m_{2}=-\bar{z} M_{k} ; \quad m_{3}=\bar{y} M_{k} ; \quad m_{4}=\overline{y z} M_{k} ; \\
& m_{5}=-\bar{x} \cdot \bar{z} M_{k} ; m_{6}=\bar{x} \cdot \bar{y} M_{k} ; \\
& c_{1}=4 c_{y} ; \quad c_{2}=2 c_{y}\left(l_{1}-l_{2}\right) ; \quad c_{3}=4 c_{z} ; \quad c_{4}=2 c_{z}\left(l_{1}-l_{2}\right) ; \\
& c_{5}=2 c_{z}\left(b_{2}-b_{1}\right) ; c_{6}=2 c_{y}\left(l_{1}^{2}+l_{2}^{2}\right) ; \\
& c_{7}=2 c_{z}\left(l_{1}^{2}+l_{2}^{2}\right) ; c_{8}=c_{z}\left(l_{1} b_{2}-l_{2} b_{2}+l_{2} b_{1}-l_{1} b_{1}\right) ; \\
& c_{9}=4 c_{y} H^{2}+2 c_{z}\left(b_{1}^{2}+b_{2}^{2}\right) .
\end{aligned}
$$

The vector of disturbing forces from the imbalance and pendulums $\{F\}$ with the selected generalized coordinates is: 


$$
\{F\}=\left\{\begin{array}{l}
M_{p} e \omega^{2} \cos \omega t+m l \sum_{k=1}^{4}\left(\ddot{\varphi}_{k} \sin \varphi_{k}+\dot{\varphi}_{k}^{2} \cos \varphi_{k}\right) ; \\
M_{p} e \omega^{2} \sin \omega t+m l \sum_{k=1}^{4}\left(-\ddot{\varphi}_{k} \cos \varphi_{k}+\dot{\varphi}_{k}^{2} \sin \varphi_{k}\right) ; \\
(A-C) \delta \omega^{2} \cos (\omega t-\varepsilon)+ \\
+m l a \sum_{k=1}^{4} \sigma_{k}\left(\ddot{\varphi}_{k} \sin \varphi_{k}+\dot{\varphi}_{k}^{2} \cos \varphi_{k}\right) ; \\
(A-C) \delta \omega^{2} \sin (\omega t-\varepsilon)+ \\
m l a \sum_{k=1}^{4} \sigma_{k}\left(-\ddot{\varphi}_{k} \cos \varphi_{k}+\dot{\varphi}_{k}^{2} \sin \varphi_{k}\right) ; \\
0
\end{array}\right\}
$$

where $\sigma_{\mathrm{k}}=1$ for $k=1,2$ and $\sigma_{\mathrm{k}}=-1$ or $k=3,4$.

\section{DERIVATION OF FORMULAS FOR SELECTING THE PARAMETERS OF THE PENDULUM AUTO-BALANCER}

Proceeding from the fact that the forces resistant to the motion of the pendulums are small and their movement is close to the uniform rotational motion, we introduce small parameter $\mu$ in the equations (2):

$$
\ddot{\varphi}_{k}=\mu \phi_{k}\left(\varphi_{k}, \dot{\varphi} k, \ddot{y}, \ddot{z}, \ddot{\theta}, \ddot{\psi}\right),(k=\overline{1,4}),
$$

where

$$
\begin{gathered}
\phi_{k}\left(\varphi_{k}, \dot{\varphi}_{k}, \ddot{y}, \ddot{z}, \ddot{\theta}, \ddot{\psi}\right)=\left[\ddot{y} \sin \varphi_{k}-\ddot{z} \cos \varphi_{k}+\right. \\
\left.+a \sigma_{k}\left(\ddot{\theta} \sin \varphi_{k}-\ddot{\psi} \cos \varphi_{k}\right)-g \cos \varphi_{k}\right] / l-\beta_{O}\left(\dot{\varphi}_{k}-\omega\right) .
\end{gathered}
$$

Assuming that the change in the generalized coordinates of the rotor and the machine is periodic, and the motion of the pendulums, as mentioned above, is close to the uniform rotational motion, we seek the solution of equations (1), (2) in the form:

$$
\begin{gathered}
y=y(\omega t) ; z=z(\omega t) ; \varphi_{x}=\varphi_{x}(\omega t) ; \theta=\theta(\omega t) ; \psi=\psi(\omega t) ; \\
\varphi_{k}=\omega t+\alpha_{k}+\mu \phi_{k}^{*}(\omega t), \quad k=\overline{1,4}
\end{gathered}
$$

Here, $q(\omega t)$ is periodic functions of time $t$ with period $2 \pi / \omega$, and $\alpha_{k}$ is unknown constants. In accordance with the method of a small parameter, supplying $\varphi_{k}$ from (4) for $\mu=0$ in (1), we obtain the generating system of equations:

$$
\begin{aligned}
& {[M]\left\{\ddot{q}_{o}\right\}+[G]\left\{\dot{q}_{o}\right\}+[K]\left\{q_{o}\right\}=} \\
& =\left\{F_{c}\right\} \cos \omega t+\left\{F_{s}\right\} \sin \omega t .
\end{aligned}
$$

Here $\left\{q_{0}\right\}=\left\{y_{0}, z_{0}, \varphi_{x 0}, \theta_{0}, \psi_{0}\right\}^{T}$

$$
\begin{aligned}
& \left\{F_{c}\right\}=\{D, N, S, W, 0\}^{T} \\
& \left\{F_{s}\right\}=\{-N, D,-W, S, 0\}^{T} \\
& D=M e \omega^{2}+m l \omega^{2}\left(\cos \alpha_{1}+\cos \alpha_{2}+\cos \alpha_{3}+\cos \alpha_{4}\right) \\
& N=m l \omega^{2}\left(\sin \alpha_{1}+\sin \alpha_{2}+\sin \alpha_{3}+\sin \alpha_{4}\right) \\
& S=(A-C) \omega^{2} \delta \cos \varepsilon+ \\
& +m l a \omega^{2}\left(\cos \alpha_{1}+\cos \alpha_{2}-\cos \alpha_{3}-\cos \alpha_{4}\right) \\
& N=-(A-C) \omega^{2} \delta \sin \varepsilon+ \\
& +m l \omega^{2}\left(\sin \alpha_{1}+\sin \alpha_{2}-\sin \alpha_{3}-\sin \alpha_{4}\right)
\end{aligned}
$$

It is seen that the equilibration (5) will take place if $D=N=S=W=0$. Then equating the right-hand sides of (6) to zero and introducing the notation

$$
\eta=\frac{M e}{m l} ; \quad \xi=\frac{(A-C) \delta \cos \varepsilon}{m l} ; \quad \zeta=\frac{(A-C) \delta \sin \varepsilon}{m l},
$$

we obtain the following system of equations for determining the unknown constants $\alpha_{k}$ :

$$
\begin{aligned}
& \cos \alpha_{1}+\cos \alpha_{2}+\cos \alpha_{3}+\cos \alpha_{4}=-\eta \\
& \sin \alpha_{1}+\sin \alpha_{2}+\sin \alpha_{3}+\sin \alpha_{4}=0 ; \\
& \cos \alpha_{1}+\cos \alpha_{2}-\cos \alpha_{3}-\cos \alpha_{4}=-\xi / a ; \\
& \sin \alpha_{1}+\sin \alpha_{2}-\sin \alpha_{3}-\sin \alpha_{4}=\zeta / a .
\end{aligned}
$$

Among the set of solutions of trigonometric equations (7) the following will be the main ones:

$$
\begin{aligned}
& \alpha_{1}=\pi-\operatorname{arctg}\left[\frac{\zeta}{\eta a+\xi}\right]-\arccos \left[\frac{1}{4 a} \sqrt{\zeta^{2}+(\eta a+\xi)^{2}}\right] \\
& \alpha_{2}=-\pi-\operatorname{arctg}\left[\frac{\zeta}{\eta a+\xi}\right]+\arccos \left[\frac{1}{4 a} \sqrt{\zeta^{2}+(\eta a+\xi)^{2}}\right] \\
& \alpha_{3}=\pi+\operatorname{arctg}\left[\frac{\zeta}{\eta a-\xi}\right]-\arccos \left[\frac{1}{4 a} \sqrt{\zeta^{2}+(\eta a-\xi)^{2}}\right] \\
& \alpha_{4}=-\pi+\operatorname{arctg}\left[\frac{\zeta}{\eta a-\xi}\right]+\arccos \left[\frac{1}{4 a} \sqrt{\zeta^{2}+(\eta a-\xi)^{2}}\right]
\end{aligned}
$$

From (8), we can obtain conditions for choosing the parameters of the auto- balancer, starting from the fact that the cosine of any angle can only be less than or equal to one.

$$
[(A-C) \delta \sin \varepsilon]^{2}+\left[M_{p} e a+(A-C) \delta \cos \varepsilon\right]^{2} \leq(4 m l a)^{2}
$$


$[(A-C) \delta \sin \varepsilon]^{2}+\left[M_{p} e a-(A-C) \delta \cos \varepsilon\right]^{2} \leq(4 m l a)^{2}$.

\section{DETERMINATION OF THE STABILITY CONDITIONS OF THE ROTOR AUTO-BALANCER INSTALLED IN THE CASING}

Finding periodic solutions of the generating equations in the form:

$$
q_{o i}=a_{i} \cos \omega t+b_{i} \sin \omega t, i=\overline{1,5},
$$

we arrive at a system of ten linear algebraic equations with respect to $a_{i}, b_{i}$. In the matrix form, these equations are written as follows:

$$
[A]\left\{q^{*}\right\}=\left\{F_{o}\right\},
$$

where $\left\{q^{*}\right\}=\left\{a_{1}, b_{1}, \ldots, a_{5}, b_{5}\right\}^{T}$

$\left\{F_{o}\right\}=\{D,-N, N, D, S,-W, W, S, 0,0\}^{T}$.

The non-zero elements of matrix $A$ have the form:

$a_{11}=a_{22}=c_{1}-\omega^{2} M^{*} ; \quad a_{33}=a_{44}=c_{3}-\omega^{2} M^{*} ;$

$a_{55}=a_{66}=c_{6}-\omega^{2} I_{z}^{*} ; \quad a_{77}=a_{88}=c_{7}-\omega^{2} I_{y}^{*} ;$

$a_{99}=a_{10,10}=c_{9}-\omega^{2} I_{x}^{*} ;$

$a_{15}=a_{26}=a_{51}=a_{62}=c_{2}-\omega^{2} m_{1} ;$

$a_{58}=a_{85}=C \omega^{2} ; a_{67}=a_{76}=-C \omega^{2}$;

$a_{37}=a_{48}=a_{73}=a_{84}=c_{4}-\omega^{2} m_{1}$;

$a_{19}=a_{2,10}=a_{91}=a_{10,2}=c_{1} H-\omega^{2} m_{2}$;

$a_{39}=a_{4,10}=a_{93}=a_{10,4}=c_{5}-\omega^{2} m_{3} ;$

$a_{57}=a_{68}=a_{75}=a_{86}=-\omega^{2} m_{4}$;

$a_{59}=a_{6,10}=a_{95}=a_{10,6}=c_{2} H-\omega^{2} m_{5} ;$

$a_{79}=a_{8,10}=a_{97}=a_{10,8}=c_{8}-\omega^{2} m_{6}$.

For sufficiently small $\mu$, periodic solutions of the generating system of equations depending on constants $\alpha_{k}$ only correspond to asymptotically stable periodic solutions of the original equations if for constants $\alpha_{k}$, that satisfy the equations:

$$
P_{s}\left(\alpha_{1}, \ldots, \alpha_{k}\right)=0, s=\overline{1, k}
$$

the condition is fulfilled, which consists in the requirement that the real parts of all roots of the algebraic equation are to be negative:

$$
\left|\frac{\partial P_{s}}{\partial \alpha_{j}}-\sigma_{s j} \lambda\right|=0
$$

$s, j=\overline{1, k}$

Here $P_{s}\left(\alpha_{1}, \alpha_{2}, \ldots, \alpha_{k}\right)$ are generating functions, and $\sigma_{s j}$ is the Kronecker symbol. Generating functions $P_{s}\left(\alpha_{1}, \alpha_{2}, \ldots, \alpha_{k}\right)$ are found after substituting $q_{o i}$ and $\varphi_{k o}=\omega t+\alpha_{k}$ into the righthand sides of (3) and averaging the obtained expressions:

$$
P_{s}\left(\alpha_{1}, \ldots, \alpha_{k}\right)=\frac{\omega}{2 \pi} \int_{0}^{\frac{2 \pi}{\omega}} \phi_{k}\left(\varphi_{k o}, \ddot{y}_{o}, \ddot{z}_{o}, \ddot{\theta}_{o}, \ddot{\psi}_{o}\right) d t .
$$

In our case:

$P_{s}\left(\alpha_{1}, \alpha_{2}, \alpha_{3}, \alpha_{4}\right)=-\frac{\omega^{2}}{2 l}\left[(\tilde{A} D+\tilde{C} N+\tilde{E} S+\tilde{G} W) \sin \alpha_{k}+\right.$ $\left.+(\tilde{B} D+\tilde{K} N+\tilde{F} S+\tilde{H} W) \cos \alpha_{k}\right]$,

where

$\tilde{A}=a_{11}+a_{14}+a_{41}+a_{44}+a\left(a_{51}+a_{54}+a_{81}+a_{84}\right) ;$

$\tilde{B}=a_{21}+a_{24}-a_{31}-a_{34}+a\left(a_{61}+a_{64}-a_{71}-a_{74}\right) ;$

$\tilde{C}=-a_{12}+a_{13}-a_{42}+a_{43}+a\left(-a_{52}+a_{53}-a_{82}+a_{83}\right) ;$

$\tilde{K}=-a_{22}+a_{23}+a_{32}-a_{33}+a\left(-a_{62}+a_{63}-a_{72}+a_{73}\right)$;

$\tilde{E}=a_{15}+a_{18}+a_{45}+a_{48}+a\left(a_{55}+a_{58}+a_{85}+a_{88}\right)$;

$\tilde{F}=a_{25}+a_{28}-a_{35}-a_{38}+a\left(a_{65}+a_{68}-a_{75}-a_{78}\right) ;$

$\tilde{G}=-a_{16}+a_{17}-a_{46}+a_{47}+a\left(-a_{56}+a_{57}-a_{86}+a_{87}\right)$;

$\tilde{H}=-a_{26}+a_{27}+a_{36}-a_{37}+a\left(-a_{66}+a_{67}+a_{76}-a_{77}\right)$.

In these formulas, $a_{j k}$ is the elements of matrix $[A]^{-1}$, and $D, N, S, W$ are determined from (6). It is not difficult to verify that the $\alpha_{k}$ found by (8) satisfy the equations (11). The expressions obtained in the analytic form for the generating functions make it possible to find partial derivatives $\partial P_{k} / \partial \alpha_{i}$, that is, the elements of the determinant (12):

$\frac{\partial P_{k}}{\partial \alpha_{i}}=\frac{m \omega^{2}}{2}\left[\tilde{A}+a \sigma_{k} \tilde{E}\right) \sin \alpha_{k} \sin \alpha_{i}-$

$-\left(\tilde{C}+a \sigma_{k} \tilde{G}\right) \sin \alpha_{k} \cos \alpha_{i}+\left(\tilde{B}+a \sigma_{k} \tilde{F}\right) \cos \alpha_{k} \sin \alpha_{i}-$

$\left.-\left(\tilde{K}+a \sigma_{k} \tilde{H}\right) \cos \alpha_{k} \cos \alpha_{i}\right]$. 
Expanding the determinant (12), we obtain an algebraic equation of the fourth order with respect to $\lambda$. It is advisable to verify the negativity of the real parts of the roots of the equation by means of the Routh-Hurwitz criterion. Carrying out such check within the chosen range of rotor angular velocities, it is possible to determine the zones of stable operation of the auto-balancing device.

\section{CONCLUSIONS AND RESULTS OF CALCULATION}

The calculation of the stability zones was carried out with the initial data corresponding to the data of the experimental stand: $M_{p}=11,3 \mathrm{~kg} ; M_{k}=57,3 \mathrm{~kg} ; A=0,285 \mathrm{kgm}^{2} ; C=0,007$ $\mathrm{kgm}^{2} ; \quad I_{x}=2,287 \mathrm{kgm}^{2} ; \quad I_{y}=6,33 \mathrm{kgm}^{2} ; I_{z}=8,353 \mathrm{kgm}^{2} ;$ $m=0,05 \mathrm{~m} ; c_{y}=c_{z}=1,85 \cdot 10^{4} \mathrm{H} / \mathrm{m} ; e=0,1 \mathrm{~mm} ; \delta=0,0005$ $\mathrm{rad} ; \quad \varepsilon=0 ; \quad l=0,08 \mathrm{~m} ; \quad b_{1}=b_{2}=0,37 \mathrm{~m} ; \quad l_{1}=0,54 \mathrm{~m} ;$ $l_{2}=0,36 \mathrm{~m} ; \quad H=0,2 \mathrm{~m} ; \quad \bar{x}=0,2 \mathrm{~m} ; \quad \bar{y}=0 ; \quad \bar{z}=-0,1 \mathrm{~m} ;$ $a_{1}=a_{4}=0,28 m ; a_{2}=a_{3}=0,25 \mathrm{~m},(a=0,265 \mathrm{~m} ;)$. Based on the results of the calculation, the resonant frequencies of the selected dynamic model are found: $\omega_{1}=30,2 \mathrm{rad} / \mathrm{s} ; \omega_{2}=32,5$ $\mathrm{rad} / \mathrm{s} ; \omega_{3}=41,8 \mathrm{rad} / \mathrm{s} ; \omega_{4}=58,8 \mathrm{rad} / \mathrm{s} ; \omega_{5}=59,4 \mathrm{rad} / \mathrm{s}$.

The analysis of frequencies and vibration modes showed that linear oscillations in horizontal and vertical directions predominate at frequencies $\omega_{1}$ and $\omega_{2}$. At frequency $\omega_{3}=41.8$ $\mathrm{rad} / \mathrm{s}$, angular oscillations around the $z$ axis occur, at a frequency $\omega_{4}=58.8 \mathrm{rad} / \mathrm{s}$, oscillations about the $x$ axis, and at $\omega_{5}=59.4 \mathrm{rad} / \mathrm{s}-$ around the $y$ axis. Fig. 2 shows the zones of stability of the auto-balancer, which are shaded. It can be seen that the stable operation of the auto-balancer is possible in two bands at $41.8<\Omega<49.4 \mathrm{rad} / \mathrm{s}$ and at $\Omega>59.4 \mathrm{rad} / \mathrm{s}$. And the last zone for this dynamic model is not bound from above. The calculations also showed that there is one more stability zone $(58.8<\Omega<59.1 \mathrm{rad} / \mathrm{s})$, but this zone is so small that it is difficult to depict it in the figure and it has no practical value.

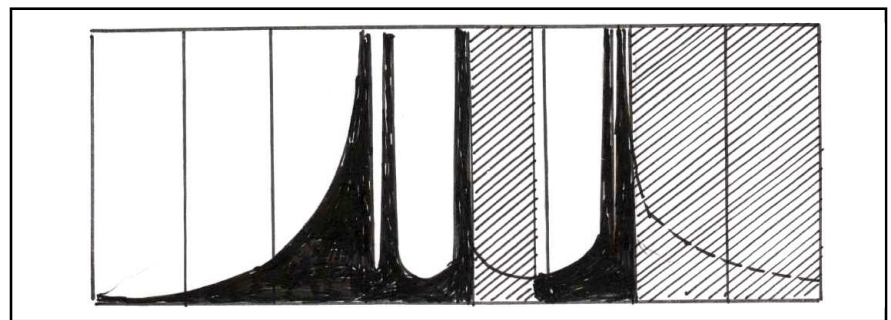

Fig. 2. Resonant zones of the rotor in the housing on elastic supports and zones of stable operation of auto-balancers (shaded).

The results of calculating the auto-balancer for the dynamic rotor model installed in the housing (Figure 1) show that the stable operation of auto-balancing devices can be achieved by the shock absorption of the housing, which does not contradict the generalized principle of auto-balancing [3]. Another important conclusion that can be drawn from the results of the research is that, by introducing the shock absorption of the housing, it is possible to increase the number of zones for the stable operation of the auto-balancer.

\section{References}

[1] A.A. Gusarov, Automatic balancing devices of direct action. Moscow: Nauka Publ., 2002, p.120.

[2] I.I. Blechman, Synchronization of dynamical systems. Moscow: Nauka Publ., 1971, pp. 896.

[3] I.I. Blechman, Vibration mechanics. Moscow: Fizmatlit Publ., 1994, pp. 400.

[4] V.P. Nesterenko, Automatic balancing of rotors, instruments and machines with many degrees of freedom. In M. P. Shumsky(ed.). Tomsk: Tomsk Polytechnic Institute, 1985, pp. 83.

[5] A.I. Artyunin, "General method for calculating auto-balancing devices for rotors of machines with a large number of degrees of freedom". Modern technologies. System analysis. Modeling. Irkutsk, vol. 3, pp. 69-72, 2011.

[6] G. B. Filimonikhin, Equilibrium and vibration protection of rotors with auto-balancers with solid corrective cargoes. Monograph (on the specialty 05.02.09 - Dynamics and strength of machines). Ministry of Education and Science of Ukraine, Kirovograd. National tech. un. Kirovograd, 2004

[7] V.G. Bykov, "Non-stationary modes of motion of a statically unbalanced rotor with an auto-balancing mechanism". Bulletin of St Petersburg University. Mathematics. Mechanics. Astronomy, vol. 3, pp. 89-96, 2010.

[8] V.G. Bykov, "Autobalance of a rigid rotor in viscoelastic orthotropic supports". Bulletin of St. Petersburg State University, Ser. 1, Issue 2, pp. 82-91, 2013

[9] K.V. Serebrennikov, Peculiarities of the Dynamics of Rotary Systems with Pendulum Autobalancers. Thesis Abstract on the Obtaining the Scientific Degree of Ph.D. (Engineering). Irkutsk, 2014, p.18.

[10] G.R. Ziyakayev, Some questions of the dynamics of rotor systems with pendulum self-balancing devices. The dissertation author's abstract on competition of a scientific degree of Ph.D. (Engineering). Tomsk: Polytechnic University, 2009, p. 19.

[11] V.A. Dubovik, G.R. Ziyakayev, "The main motion of a twopendulum auto balance on a flexible shaft with elastic supports". Izvestia of Tomsk Polytechnic. un-ty. Mathematics and mechanics. Physics, vol. 317-2, pp. 37-39, 2010.

[12] L. Sperling, B. Ryzhik, Ch. Linz, H. Duckstein, "Simulation of two simple automatic balancing of a rigid rotor". Mathematics and Computers in Simulation, vol. 58, no. 4-6, pp. 351-365, 2002.

[13] D.J. Rodrigues, A.R. Champneys, M.I. Friswell, "Automatic twoplane balancing for rigid rotors". International Journal of Mon-Linear Mechanics, vol. 43, iss. 6, pp. 527-541, 2008.

[14] D.J. Rodrigues, Champneys, M.I. Friswell, R.E. Wilson, "Two-plane automatic balancing: a symmetry breaking analysis". International Journal of Mon-Linear Mechanics., vol. 46, iss. 9, pp. 1139-1154, 2011.

[15] J.N. Bolton, Single-and dual-plane automatic balancing of an elastically mounted cylindrical rotor with considerations of coulomb friction and gravity. Dr. Diss. Blacksburg, Virginia, 2010, p. 317.

[16] A.N. Nikiforov, "The state of the problem of balancing rotors". Bulletin of scientific and technical development, vol. 4 (68), pp. 2028, 2013.

[17] A.I. Artyunin, G.G. Alkhunsaev, Zh.B. Sushkeev, "Auto-balance of rotors in hulls on elastic stops". Modern technologies. System analysis. Modeling, vol. 6, pp. 38-41, 2006.

[18] G.B. Filimonikhin, V.V. Goncharov, "Balancing the auto-balancer of the rotor in an elastically viscous fixed body that performs spatial motion". Bulletin of the Tomsk Polytechnic University, vol. 325-2, pp. 41-49, 2014. 